\title{
Vasohibin-2 promotes proliferation in human breast cancer cells via upregulation of fibroblast growth factor-2 and growth/differentiation factor-15 expression
}

\author{
MIN TU ${ }^{*}$, XIAN LIU ${ }^{1 *}$, BEI HAN ${ }^{2}$, QIANQIAN GE ${ }^{1}$, ZHANJUN LI $^{1}$, ZIPENG LU ${ }^{1}$, JISHU WEI ${ }^{1}$, GUOXIN SONG ${ }^{3}$, \\ BAOBAO CAI $^{1}$, NAN LV ${ }^{1}$, KUIRONG JIANG ${ }^{1}$, SHUI WANG ${ }^{1}$, YI MIAO ${ }^{1}$ and WENTAO GAO ${ }^{1}$ \\ ${ }^{1}$ Department of General Surgery, The First Affiliated Hospital of Nanjing Medical University, Nanjing, Jiangsu 210029; \\ ${ }^{2}$ Department of Endocrinology, Nanjing Children's Hospital Affiliated to Nanjing Medical University, Nanjing, Jiangsu 210008; \\ ${ }^{3}$ Department of Pathology, The First Affiliated Hospital of Nanjing Medical University, Nanjing, Jiangsu 210029, P.R. China
}

Received February 21, 2014; Accepted June 5, 2014

DOI: $10.3892 / \mathrm{mmr} .2014 .2317$

\begin{abstract}
Vasohibin-2 (VASH2) is an angiogenic factor, and has been previously reported to be a cancer-related gene, with cytoplasmic and karyotypic forms. In the current study VASH2 expression in human breast cancer tissue and adjacent non-cancerous tissue was investigated with immunohistochemistry. MCF-7 and BT474 human breast cancer cells were transfected with lentiviral constructs to generate in vitro VASH2 overexpression and knockdown models. In addition, BALB/cA nude mice were inoculated subcutaneously with transfected cells to generate in vivo models of VASH2 overexpression and knockdown. The effect of VASH2 on cell proliferation was investigated using a bromodeoxyuridine assay in vitro and immunohistochemistry of Ki67 in xenograft tumors. Growth factors were investigated using a human growth factor array, and certain factors were further confirmed by an immunoblot. The results indicated that the expression level of cytoplasmic VASH2 was higher in breast cancer tissues with a Ki67 (a proliferation marker) level of $\geq 14 \%$, compared with tissues with a Ki67 level of $<14 \%$. VASH2 induced proliferation in vitro and in vivo. Four growth factors activated by VASH2 were identified as follows: Fibroblast growth factor 2 (FGF2), growth/differentiation factor-15 (GDF15), insulin-like growth factor-binding protein (IGFBP)3 and IGFBP6. FGF2 and GDF15 may contribute to VASH2-induced proliferation. The current
\end{abstract}

Correspondence to: Professor Yi Miao or Professor Wentao Gao, Department of General Surgery, The First Affiliated Hospital of Nanjing Medical University, 300 Guangzhou Road, Nanjing, Jiangsu 210029, P.R. China

E-mail: miaoyi@njmu.edu.cn

E-mail: gao11@hotmail.com

*Contributed equally

Key words: vasohibin-2, breast cancer, proliferation, growth factor study identified a novel role for VASH2 in human breast cancer, and this knowledge suggests that VASH2 may be a novel target in breast cancer treatment.

\section{Introduction}

The vasohibin (VASH) family consists of two members, VASH1 and VASH2 (1). VASH1 was initially identified as a regulator of negative feedback in angiogenesis induced by vascular endothelial growth factor (VEGF) or fibroblast growth factor 2 (FGF2) (2,3). VASH2 is a VASH1 homolog expressed in mononuclear cells that has been demonstrated to act as an angiogenesis stimulator in a mouse model of hypoxia-induced subcutaneous angiogenesis (3). VASH2 is also involved in the proliferation of hepatic (4) and ovarian $(5,6)$ cancer.

It was previously demonstrated that there are two types of VASH2: Nuclear and cytoplasmic (7). In the present study, the focus was on cytoplasmic VASH2, thus all subsequent mention of VASH2 refers to the cytoplasmic form. VASH2 expression was investigated in human breast cancer in the current study; rabbit polyclonal anti-human VASH2 antibodies were produced and successfully used in immunoblotting and immunohistochemical analysis (7).

In the present study, VASH2 expression levels were indicated to be higher in grade 3 vs. grade 1-2 tissues, and in tissues with a level of $\mathrm{Ki} 67 \geq 14 \%$. Ki67 is a marker for breast cancer proliferation. It was hypothesized that VASH2 is associated with cell proliferation in breast cancer, and in order to investigate the proliferative function of VASH2 in breast cancer cells and the underlying mechanism, VASH2 overexpression and knockdown in vitro and in vivo models were established. VASH2 produced a significant proliferative effect in vitro and in vivo. Human growth factor array demonstrated that VASH2 promoted proliferation in breast cancer cells via the upregulation of FGF2 and growth/differentiation factor-15 (GDF15) expression. The present study identified a novel role for VASH2 in human breast cancer, and this knowledge may lead to the possibility of VASH2 as a novel target in breast cancer treatment. 


\section{Materials and methods}

Clinical samples. Human breast cancer tissue and adjacent non-cancerous tissue were obtained from 99 patients who underwent surgical resection at The First Affiliated Hospital of Nanjing Medical University (Nanjing, China) in accordance with institutional policy. All patients provided written informed consent.

Animals. Five-week-old female BALB/cA-nu (nu/nu) nude mice were obtained from Vital River Laboratories (Beijing, China). The Animal Care and Use Subcommittee of Nanjing Medical University approved all experimental procedures, which were performed in accordance with the standards established by the 1964 Declaration of Helsinki and its later amendments. Animals were sacrificed using pure carbon dioxide.

Cell culture. The MCF-7 human breast cancer cell line was obtained from the Shanghai Cell Bank (Type Culture Collection Committee, Chinese Academy of Sciences, Shanghai, China) and cultured according to the manufacturer's instructions. The BT474 human breast cancer cell line was provided by Professor Shui Wang of the Department of General Surgery, The First Affiliated Hospital of Nanjing Medical University (Nanjing, China) and cultured in complete Dulbecco's modified Eagle's medium (Gibco, Carlsbad, CA, USA) supplemented with 10\% fetal bovine serum (Gibco). Cells were cultivated in a humidified $5 \% \mathrm{CO}_{2}$ incubator at $37^{\circ} \mathrm{C}$.

Plasmid construction and lentivirus packaging. Lentiviral (Lv) constructs were designed to induce VASH2 overexpression and knockdown as previously described (4). MCF-7 cells were stably transfected with Lv-CMV-VASH2 for VASH2 overexpression and termed MCF7-VASH2; MCF-7 cells stably transfected with Lv-CMV-enhanced green fluorescent protein (EGFP) for VASH2 knockdown were termed MCF7-EGFP; BT474 cells stably transfected with VASH2-targeting short hairpin RNA (shRNA) lentivirus for VASH2 knockdown were termed BT474-shVASH2; and BT474 cells stably transfected with scrambled shRNA lentivirus as controls were termed BT474-scramble.

Immunoblotting. Whole cell lysates were prepared in radioimmunoprecipitation assay buffer (Beyotime, Nantong, China) and blotted using the following primary antibodies: Rabbit polyclonal anti-VASH2 [prepared as described in (7)]; rabbit polyclonal anti-FGF2 (Sigma-Aldrich, St. Louis, MO, USA); goat polyclonal anti-GDF15 (Santa Cruz Biotechnology, Santa Cruz, CA, USA); and mouse monoclonal anti-GAPDH (Beyotime, Nantong, China). The secondary antibodies used for detection were horseradish peroxidase (HRP)-conjugated goat anti-mouse immunoglobulin $\mathrm{G}(\mathrm{IgG})$ (CWbio, Shanghai, China) and HRP-conjugated donkey anti-rabbit IgG (CWbio).

Immunohistochemistry. Immunohistochemical staining of the clinical samples was performed as previously described (7). Xenograft tumors were harvested from mice and stained with the following primary antibodies: Rabbit polyclonal anti-VASH2 [prepared as previously described (7)], rabbit polyclonal anti-estrogen receptor (ER) $\alpha$ and Ki67 (Maixin
Biotech, Fuzhou, China), and mouse monoclonal antibodies targeting the progesterone receptor (PR) and human epidermal growth factor receptor 2 (HER2; Maixin Biotech). VASH2 staining intensity was classified as weak or strong. ER $\alpha, P R$ and HER2 staining were classified as positive or negative. HER $2^{++++}$was also classified as negative; only HER $2^{+++}$was classified as positive. Ki67 staining was classified as $<14 \%$ and $\geq 14 \%$. VASH2 staining intensity was classified as Low (negative or weak staining) and high (middle or strong staining).

In vivo tumorigenesis. MCF7-EGFP or MCF7-VASH2 cells $\left(2 \times 10^{6}\right)$ were bilaterally injected subcutaneously into the flanks of eight mice. Eighty days later, the mice were sacrificed and the xenograft tumors harvested. In addition, BT474-scramble or BT474-shVASH2 cells $\left(1 \times 10^{6}\right)$ were bilaterally injected subcutaneously into the flanks of seven mice and the xenograft tumors were harvested at 60 days post-inoculation. Tumor volume was calculated as follows: (Width ${ }^{2} \mathrm{x}$ length)/2.

Bromodeoxyuridine (BrdU) proliferation assay. MCF7-EGFP, MCF7-VASH2 (2x10 cells/well), BT474-scramble and BT474-shVASH 2 cells $\left(3 \times 10^{3}\right.$ cells/well $)$ were seeded in 96-well tissue culture plates. Eight wells were used for each cell type. At $48 \mathrm{~h}$ following seeding, cell proliferation enzyme-linked immunosorbent assay (ELISA) using a BrdU kit (cat no. 11647229001; Roche, Mannheim, Germany) was performed according to the manufacturer's instructions.

Antibody array. Quantitative sandwich-based antibody array (\#QAH-GF-1; RayBiotech, Guangzhou, China) was used to detect 40 human growth factors in lysates of the MCF7-EGFP, MCF7-VASH2, BT474-scramble and BT474-shVASH2 cells. All detection services were provided by RayBiotech (Norcross, GA, USA). Each antibody produced four dots, and the averages of the median signal intensities were used for all calculations. Fold change of $>1.5$ or $<0.66$ compared with controls was selected as the distinction between overexpression or knockdown, respectively.

Statistical analysis. Statistical analysis was performed using SPSS, version 13.0 (SPSS, Inc., Chicago, IL, USA). Comparisons between treated and control groups were conducted using Student's t-test, and $\mathrm{P}<0.05$ was considered to indicate a statistically significant difference. Pearson's $\chi^{2}$ test was used to compare rates of the data in Table I.

\section{Results}

VASH2 staining and the clinicopathological characteristics of breast cancer. VASH2 expression levels in 99 human breast cancer tissue samples were assessed by immunohistochemical analysis. Table I shows the association between VASH2 staining and the clinicopathological characteristics of breast cancer. VASH2 expression was high in 70/99 (70.7\%) breast cancer tissues. VASH2 staining was generally higher in grade 3 tissues and those with Ki67 $\geq 14 \%$ (Pearson $\chi^{2}, P<0.001$ ). These findings indicate that VASH2 may promote proliferation in human breast cancer cells. Fig. 1 displays the representative images of high and low VASH2 expression levels in human breast cancer tissues. 
Table I. Association of VASH2 staining with clinicopathological characteristics of breast cancer.

\begin{tabular}{|c|c|c|c|c|}
\hline \multirow{2}{*}{$\begin{array}{l}\text { Clinicopathologic } \\
\text { characteristic }\end{array}$} & \multirow{2}{*}{$\begin{array}{l}\text { No. of } \\
\text { patients }\end{array}$} & \multicolumn{2}{|c|}{ VASH2 staining } & \multirow[b]{2}{*}{ P-value } \\
\hline & & Low $(\%)$ & High (\%) & \\
\hline Age & & & & 0.344 \\
\hline$<45$ & 27 & $6(22.3)$ & $21(77.7)$ & \\
\hline$\geq 45$ & 72 & $23(31.9)$ & $49(68.1)$ & \\
\hline Pathological stage & & & & 0.299 \\
\hline $\mathrm{T} 1$ & 50 & $17(34.0)$ & $33(66.0)$ & \\
\hline $\mathrm{T} 2-4$ & 49 & $12(24.5)$ & $37(75.5)$ & \\
\hline Tumor grade & & & & $<0.001$ \\
\hline G1-2 & 66 & $27(40.9)$ & $39(59.1)$ & \\
\hline G3 & 33 & $2(6.1)$ & $31(93.9)$ & \\
\hline Node status & & & & 0.952 \\
\hline Negative & 61 & $18(29.5)$ & $43(70.5)$ & \\
\hline Positive & 38 & $11(28.9)$ & $27(71.1)$ & \\
\hline ER $\alpha$ status & & & & 0.066 \\
\hline Negative & 34 & $6(17.6)$ & $28(82.4)$ & \\
\hline Positive & 65 & $23(35.4)$ & $42(64.6)$ & \\
\hline PR status & & & & 0.734 \\
\hline Negative & 47 & $13(27.7)$ & $34(72.3)$ & \\
\hline Positive & 52 & $16(30.8)$ & $36(69.2)$ & \\
\hline HER-2 status & & & & 0.988 \\
\hline Negative & 75 & $22(29.3)$ & $53(70.7)$ & \\
\hline Positive & 24 & $7(29.2)$ & $17(70.8)$ & \\
\hline Ki67 status & & & & $<0.001$ \\
\hline$<14 \%$ & 28 & $19(67.9)$ & $09(32.1)$ & \\
\hline$\geq 14 \%$ & 71 & $10(14.1)$ & $61(85.9)$ & \\
\hline Total & 99 & $29(29.3)$ & $70(70.7)$ & \\
\hline
\end{tabular}

VASH2, vasohibin-2; ER, estrogen receptor; HER, human epidermal growth factor receptor. VASH2 staining: Low, negative or weak staining; High, middle or strong staining.

VASH2 promotes proliferation in human breast cancer cells in vitro and in vivo. The VASH2 expression levels in VASH2-overexpressing (MCF7-VASH2) and VASH2 knockdown cells (BT474-shVASH2) were confirmed using immunoblotting (Fig. 2), indicating the successful establishment of in vitro models of VASH2 overexpression and knockdown. The proliferative function of VASH2 was investigated in vitro using cell proliferation ELISAs. Results indicated that the optical density at $450 \mathrm{~nm}$ (OD450) of MCF7-VASH2 cells was significantly higher than that of MCF7-EGFP cells, while the OD450 of BT474-shVASH2 cells was significantly lower than that of BT474-scramble cells (Fig. 3A, $\mathrm{P}<0.05$ ). These data indicate that VASH2 induced cell proliferation in vitro.

MCF7-EGFP, MCF7-VASH2, BT474-scramble or BT474-shVASH2 cells were injected into the flanks of nude mice. At 80 days post-inoculation, mice that had been injected with MCF7-VASH2 cells had developed significantly larger tumors than mice injected with MCF7-EGFP cells (Fig. 3B, $\mathrm{P}<0.05$ ). At 60 days post-inoculation, mice that had been injected with BT474-shVASH2 cells had developed significantly smaller tumors than mice injected with
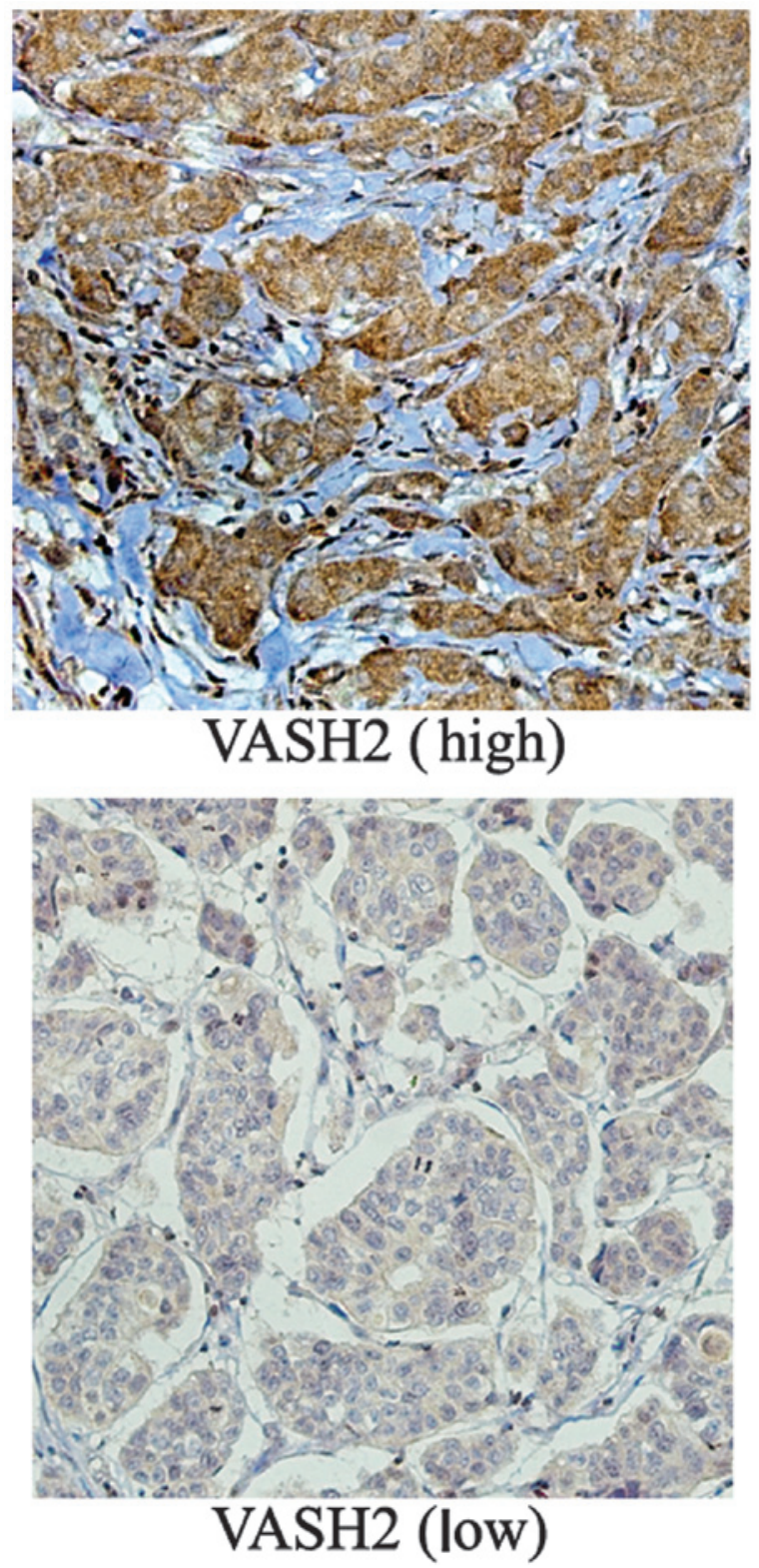

Figure 1. Representative images of VASH2 staining in human breast cancer tissues. Magnification, x200. VASH2, vasohibin-2.

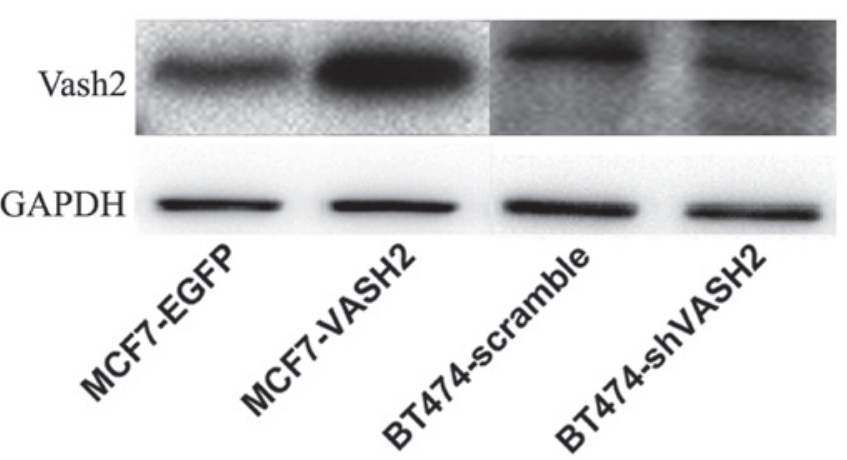

Figure 2. VASH2 expression in stably transfected MCF-7 and BT474 cells. MCF-7 cells were transfected with a vector expressing EGFP (MCF7-EGFP) or VASH2 (MCF7-VASH2); BT474 cells were transduced with scrambled shRNA (BT474-scramble) or VASH2-targeting shRNA (BT474-shVASH2). The level of VASH2 protein in each cell type was assessed by immunoblotting. GAPDH, glyceraldehyde-3-phosphate dehydrogenase; VASH2, vasohibin-2; EGFP, enhanced green fluorescent protein; shRNA, short hairpin RNA. 
A

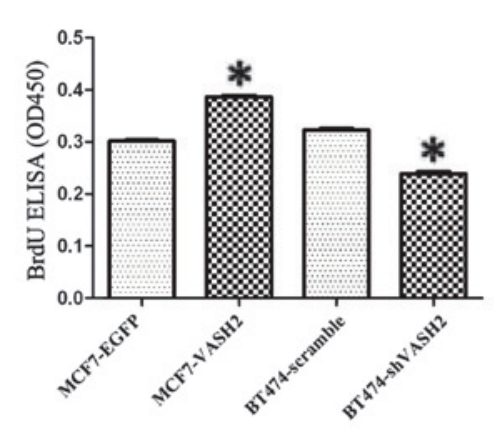

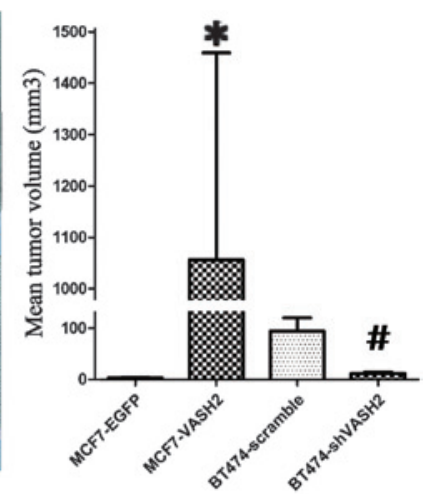

\section{C}
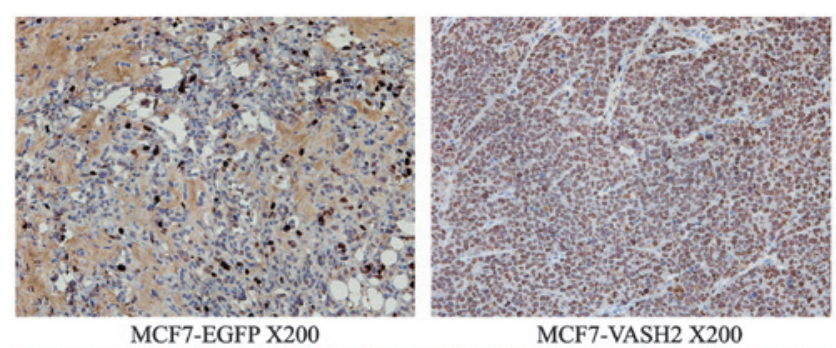

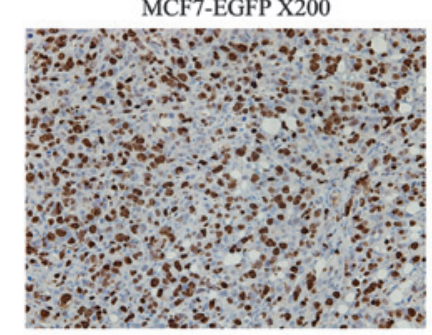

BT474-scramble X200

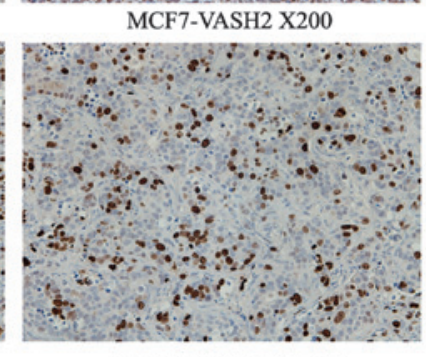

BT474-shVASH2 X200

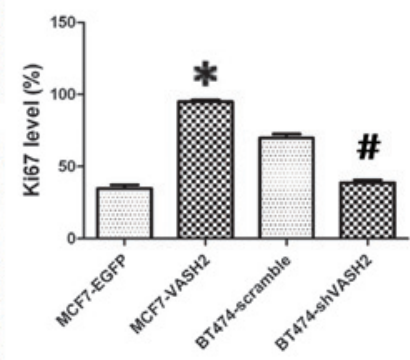

Figure 3. VASH2 induced proliferation in human breast cancer cells in vitro and in vivo. (A) In vitro effects of VASH2 on cell proliferation measured by BrdU incorporation, which was measured using ELISA. Absorbance was read at $450 \mathrm{~nm}\left({ }^{*} \mathrm{P}<0.05\right.$, $\left.\mathrm{n}=8\right)$. (B) Xenograft tumors from mice injected subcutaneously with MCF7-EGFP, MCF7-VASH2, BT474-scramble or BT474-shVASH2 cells. The data are presented as the mean \pm standard error of tumor volume of each group. MCF7-EGFP $\left(2.8 \pm 1.1 \mathrm{~mm}^{3}\right)$ vs. MCF7-VASH2 $\left(1057.0 \pm 402.8 \mathrm{~mm}^{3}\right),{ }^{*} \mathrm{P}<0.05, \mathrm{n}=8$; BT474-scramble $\left(94.4 \pm 25.5 \mathrm{~mm}^{3}\right)$ vs. BT474-shVASH2 $\left(11.3 \pm 3.3 \mathrm{~mm}^{3}\right),{ }^{\#} \mathrm{P}<0.05, \mathrm{n}=7$. (C) Immunohistochemistry of Ki67 in xenograft tumors. The data presented are the average Ki67 level \pm standard error $(\%)$ of tumors for each group. MCF7-EGFP (34.8 \pm 2.5$)$ vs. MCF7-VASH2 (95.0 \pm 1.2$),{ }^{*} \mathrm{P}<0.05$; BT474-scramble (69.8 \pm 2.8$)$ vs. BT474-shVASH2 (33.8 \pm 1.8$),{ }^{*} \mathrm{P}<0.05$. BrdU, bromodeoxyuridine; OD, optical density; EGFP, enhanced green fluorescent protein; VASH2, vasohibin-2.

BT474-scramble cells (Fig. 3B, P<0.05). The levels of Ki67 staining in MCF7-VASH2 xenograft tumors were significantly higher than in MCF7-EGFP xenograft tumors (Fig. 3C, P<0.05), and the levels in BT474-shVASH2 xenograft tumors were significantly lower than in BT474-scramble xenograft tumors (Fig. 3C, P<0.05). These findings indicate that VASH2 also induces proliferation in vivo.

VASH2 induces FGF2, GDF15, insulin-like growth factor-binding protein (IGFBP) 3 and IGFBP6 expression. Using sandwich-based antibody array, 40 human growth factors were detected in MCF7-EGFP, MCF7-VASH2, BT474-scramble and BT474-shVASH2 cell lysate samples. Table II denotes the growth factor array results. A fold change of $>1.5$ or $<0.66$ compared with controls was selected as the definition of up- and downregulation, respectively. FGF2, GDF15, IGFBP3 and IGFBP6 expression levels were elevated in the VASH2-overexpressing MCF7-VASH2 cells and were reduced in the VASH2-knockdown BT474-shVASH2 cells; this indicates that they may have contributed to the specific reaction induced by VASH2 (Fig. 4). The protein expression levels of FGF2 and GDF15 were then detected by immunoblot, which confirmed the results of the antibody array (Fig. 4D). Androgen receptor, brain-derived neurotrophic factor, bone morphogenetic protein-4, epidermal growth factor (EGF), heparin-binding EGF, IGFBP2, and VEGF receptor-2 fold changes were also $>1.5$ (Table II) in the VASH2-overexpressing MCF7-VASH2 cells, but were not altered in VASH2-knockdown BT474-shVASH2 cells. Notably, the fold changes for platelet-derived growth factor-AA and placental growth factor in the VASH2-overexpressing and knockdown cells were $<0.66$ (Table II), indicating that they may have contributed to a non-specific reaction. These data suggest that VASH2 upregulates FGF2, GDF15, IGFBP3 and IGFBP6 expression.

\section{Discussion}

VASH2 is involved in tumor proliferation $(4,5)$. Rabbit anti-human VASH2 polyclonal antibodies were generated and used in immunohistochemical analysis of VASH2 expression 
Table II. Human growth factor array results (\#QAH-GF-1; RayBiotech).

\begin{tabular}{|c|c|c|c|c|c|c|}
\hline $\begin{array}{l}\text { Growth } \\
\text { factor ID }\end{array}$ & MCF7-EGFP & MCF7-VASH2 & BT474-scramble & BT474-shVASH2 & $\begin{array}{c}\text { MCF7-VASH2 } \\
\text { /MCF7-EGFP } \\
\text { (fold change) }\end{array}$ & $\begin{array}{c}\text { BT474-shVASH2 } \\
\text { /BT474-scramble } \\
\text { (fold change) }\end{array}$ \\
\hline POS1 & 31828 & 31621 & 31798 & 31686 & 0.99 & 1 \\
\hline POS2 & 8172 & 8225 & 8180 & 8209 & 1.01 & 1 \\
\hline $\mathrm{AR}$ & 124 & 3345 & 154 & 135 & 26.92 & 0.88 \\
\hline BDNF & 71 & 268 & 121 & 94 & 3.8 & 0.78 \\
\hline FGF2 & 5539 & 38221 & 255 & 27 & 6.9 & 0.1 \\
\hline BMP-4 & 43 & 126 & 48 & 43 & 2.91 & 0.89 \\
\hline BMP-5 & 175 & 226 & 205 & 220 & 1.29 & 1.07 \\
\hline BMP-7 & 202 & 282 & 207 & 220 & 1.4 & 1.06 \\
\hline$\beta$-NGF & 45 & 35 & 44 & 31 & 0.78 & 0.71 \\
\hline EGF & 22 & 58 & 1037 & 909 & 2.67 & 0.88 \\
\hline EGF R & 1237 & 922 & 1024 & 629 & 0.75 & 0.61 \\
\hline EG-VEGF & 39 & 57 & 39 & 52 & 1.46 & 1.33 \\
\hline FGF-4 & 55 & 75 & 45 & 31 & 1.35 & 0.7 \\
\hline FGF-7 & 54 & 81 & 47 & 45 & 1.49 & 0.96 \\
\hline GDF-15 & 783 & 34885 & 418 & 151 & 44.54 & 0.36 \\
\hline GDNF & 110 & 113 & 42 & 62 & 1.02 & 1.48 \\
\hline $\mathrm{GH}$ & 318 & 351 & 413 & 283 & 1.1 & 0.68 \\
\hline HB-EGF & 262 & 393 & 269 & 324 & 1.5 & 1.21 \\
\hline HGF & 47 & 41 & 28 & 26 & 0.87 & 0.92 \\
\hline IGFBP-1 & 102 & 148 & 104 & 70 & 1.45 & 0.67 \\
\hline IGFBP-2 & 2357 & 5127 & 3863 & 4603 & 2.18 & 1.19 \\
\hline IGFBP-3 & 63 & 249 & 63 & 28 & 3.97 & 0.44 \\
\hline IGFBP-4 & 68 & 97 & 77 & 95 & 1.44 & 1.24 \\
\hline IGFBP-6 & 16 & 170 & 47 & 24 & 10.86 & 0.51 \\
\hline IGF-I & 36 & 53 & 30 & 22 & 1.47 & 0.72 \\
\hline Insulin & 232 & 289 & 259 & 258 & 1.25 & 1 \\
\hline MCF R & 135 & 161 & 133 & 111 & 1.19 & 0.84 \\
\hline NGF R & 121 & 137 & 126 & 137 & 1.14 & 1.09 \\
\hline NT-3 & 70 & 102 & 53 & 42 & 1.46 & 0.79 \\
\hline NT-4 & 70 & 101 & 48 & 46 & 1.45 & 0.95 \\
\hline OPG & 38 & 30 & 36 & 29 & 0.78 & 0.8 \\
\hline PDGF-AA & 299 & 195 & 134 & 56 & 0.65 & 0.42 \\
\hline PIGF & 570 & 122 & 199 & 120 & 0.21 & 0.6 \\
\hline SCF & 70 & 100 & 48 & 51 & 1.43 & 1.07 \\
\hline SCF R & 356 & 195 & 172 & 161 & 0.55 & 0.94 \\
\hline TGF $\alpha$ & 27 & 32 & 18 & 21 & 1.21 & 1.16 \\
\hline TGF $\beta 1$ & 376 & 532 & 414 & 435 & 1.41 & 1.05 \\
\hline TGF $\beta 3$ & 44 & 64 & 50 & 37 & 1.45 & 0.73 \\
\hline VEGF & 156 & 160 & 117 & 92 & 1.02 & 0.79 \\
\hline VEGF R2 & 42 & 76 & 34 & 28 & 1.81 & 0.83 \\
\hline VEGF R3 & 656 & 809 & 774 & 789 & 1.23 & 1.02 \\
\hline VEGF-D & 305 & 431 & 338 & 331 & 1.41 & 0.98 \\
\hline
\end{tabular}

EGFP, enhanced green fluorescent protein; VASH2, vasohibin-2; AR, androgen receptor; BDNF, brain-derived neurotrophic factor; FGF, fibroblast growth factor; BMP, bone morphogenetic protein; EGF, epidermal growth factor; GDF, growth/differentiation factor; HB-EGF, heparin binding-EGF; IGFBP, insulin-like growth factor-binding protein; VEGF, vascular endothelial growth factor.

levels (7). In the present study, VASH2 expression in clinical human breast cancer tissues was investigated, and significantly higher levels of VASH2 in grade 3 and $\mathrm{Ki} 67 \geq 14 \%$ breast cancer tissues were detected. Ki67 expression levels vary during the cell cycle; the levels are low during the $G_{1}$ and early $S$ phases, and high during mitosis, followed by a sharp reduction during anaphase and telophase. Ki67 cannot be detected during the $\mathrm{G}_{0}$ resting phase $(8,9)$. Ki67 inhibition leads to the arrest of cell proliferation $(10,11)$. Ki67 expression levels increase progressively from benign breast disease to ductal carcinoma in situ to invasive breast cancer (12-14). In addition, Ki67 is considered to be a good proliferation marker in clinical practice (15). In the 
A

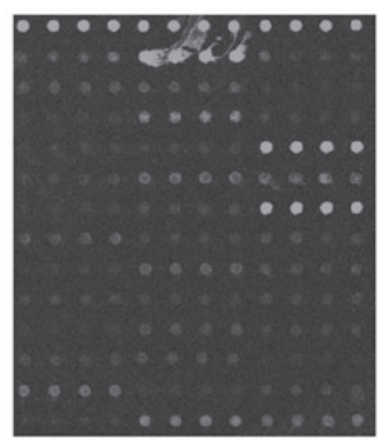

MCF7-VASH2

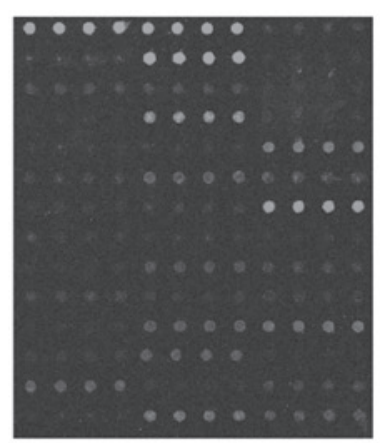

MCF7-EGFP

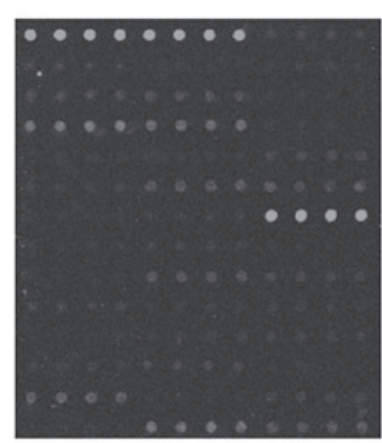

BT474-shVASH2

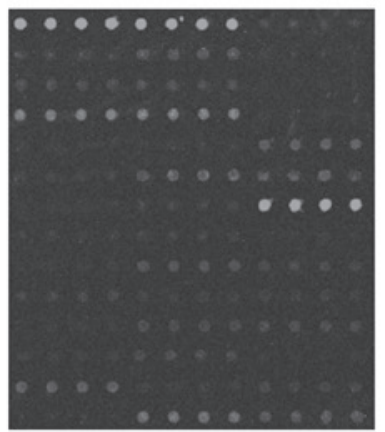

BT474-scramble
B

Growth factor ID
\begin{tabular}{|c|c|c|}
\hline \hline POS1 & POS2 & AR \\
\hline BDNF & FGF2 & BMP-4 \\
\hline BMP-5 & BMP-7 & $\beta$-NGF \\
\hline EGF & EGF R & EG-VEGF \\
\hline FGF-4 & FGF-7 & GDF-15 \\
\hline GDNF & GH & HB-EGF \\
\hline HGF & IGFBP-1 & IGFBP-2 \\
\hline IGFBP-3 & IGFBP-4 & IGFBP-6 \\
\hline IGF-I & Insulin & MCF R \\
\hline NGF R & NT-3 & NT-4 \\
\hline OPG & PDGF-AA & PIGF \\
\hline SCF & SCF R & TGF $\alpha$ \\
\hline TGF 1 1 & TGF $\beta 3$ & VEGF \\
\hline VEGF R2 & VEGF R3 & VEGF-D \\
\hline
\end{tabular}

C

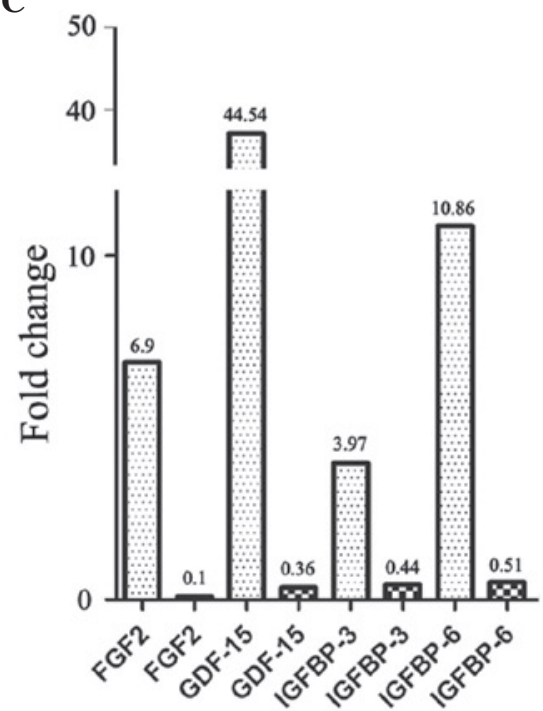

D

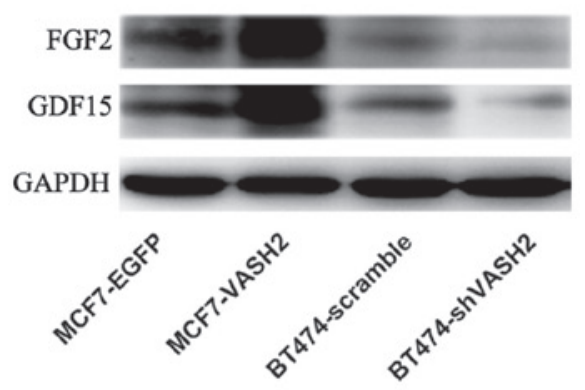

Figure 4. Human growth factor array and verification of positive hits. (A) Dots on human growth factor antibody arrays. (B) Names of the tested angiogenic factors. Growth factor fold changes of $>1.5$ in VASH2-overexpressing cells or $<0.66$ in VASH2 knockdown cells were selected as up- and downregulated, respectively (highlighted). (C) Distinct fold change of four growth factors: FGF2, GDF15, IGFBP3 and IGFBP6. (D) Immunoblot analysis of FGF2 and GDF15. VASH2; vasohibin-2; EGFP, enhanced green fluorescent protein; AR, androgen receptor; BDNF, brain-derived neurotrophic factor; FGF, fibroblast growth factor; BMP, bone morphogenetic protein; EGF, epidermal growth factor; GDF, growth/differentiation factor; HB-EGF, heparin binding-EGF; IGFBP, insulin-like growth factor-binding protein; VEGF, vascular endothelial growth factor.

current study, it was hypothesized that VASH2 is associated with cell proliferation, and to confirm the possible function of VASH2 in proliferation, in vitro and in vivo models of VASH2 overexpression and knockdown were developed. Analysis of the models indicated that VASH2 promotes the proliferation of breast cancer cells in vitro and in vivo.

Various cancer cells synthesize growth factors to which they are responsive (16), and these growth factors are important in the processes of tumor cell clonal expansion, angiogenesis, invasion and metastasis (17). It was hypothesized that VASH2 may induce proliferation via activation of growth factor expression. To confirm this, human growth factor array analysis was performed using VASH2-overexpression and knockdown in vitro models. A total of 40 common proliferation-related growth factors in four cell lysate samples (MCF7-VASH2, MCF7-EGFP, BT474-shVASH2 and BT474-scramble) were investigated. VASH2 increased the expression of four growth factors: FGF2, GDF15, IGFBP3 and IGFBP6. FGF2 (18) induces cell proliferation in various types of cancer. GDF15 serves a function in cell proliferation, apoptosis, metastasis and angiogenesis, through autocrine and paracrine signaling (19).
IGFBP3 and IGFBP6 are IGF-binding proteins that inhibit IGFs, therefore functioning as tumor suppressors $(20,21)$. However, IGFBP3 overexpression in breast cancer is linked to poor prognosis $(22,23)$. Previously, it has been reported that IGFBP3 promotes cancer cell growth via an IGF-independent manner (24). It was also reported that IGFBP6 promoted cancer cell migration in an IGF-independent manner (21). Therefore, the function of VASH2-regulated IGFBP3 and IGFBP6 expression remains unclear. It is possible that the VASH2-induced proliferation occurred via upregulation of the expression of FGF2 and GDF15.

The present study demonstrated a high level of VASH2 expression in breast cancer cells, and that VASH2 functions as an inducer of growth factor expression, promoting cell proliferation in breast cancer. In conclusion, the current study indicated that VASH2 may have potential as a novel anticancer target.

\section{Acknowledgements}

The present study was partially supported by the National Natural Science Foundation of China (81272239, 81170336, 
81172267 and 81372657), the Program for Development of Innovative Research Team in the First Affiliated Hospital of Nanjing Medical University (Jiangsu, China), the Priority Academic Development Program of Jiangsu Higher Education Institutions (PAPD, JX10231801), the Special Research Fund for Public Welfare Industry of Health (201202007), and the Graduate Education Innovation Project of Jiangsu Province (JX22013230).

\section{References}

1. Sato Y: The vasohibin family: a novel family for angiogenesis regulation. J Biochem 153: 5-11, 2013.

2. Shibuya T, Watanabe K, Yamashita H, Shimizu K, Miyashita H, Abe M, Moriya T, Ohta H, Sonoda H, Shimosegawa T, Tabayashi K and Sato Y: Isolation and characterization of vasohibin-2 as a homologue of VEGF-inducible endothelium-derived angiogenesis inhibitor vasohibin. Arterioscler Thromb Vasc Biol 26: 1051-1057, 2006.

3. Kimura H, Miyashita H, Suzuki Y, Kobayashi M, Watanabe K, Sonoda H, Ohta H, Fujiwara T, Shimosegawa T and Sato Y: Distinctive localization and opposed roles of vasohibin-1 and vasohibin-2 in the regulation of angiogenesis. Blood 113: 4810-4818, 2009.

4. Xue X, Gao W, Sun B, Xu Y, et al: Vasohibin 2 is transcriptionally activated and promotes angiogenesis in hepatocellular carcinoma. Oncogene 32: 1724-1734, 2013.

5. Takahashi Y, Koyanagi T, Suzuki Y, Saga Y, Kanomata N, Moriya T, Suzuki M and Sato Y: Vasohibin-2 expressed in human serous ovarian adenocarcinoma accelerates tumor growth by promoting angiogenesis. Mol Cancer Res 10: 1135-1146, 2012.

6. Koyanagi T, Suzuki Y, Saga Y, Machida S, Takei Y, Fujiwara H, Suzuki M and Sato Y: In vivo delivery of siRNA targeting vasohibin-2 decreases tumor angiogenesis and suppresses tumor growth in ovarian cancer. Cancer Sci 104: 1705-1710, 2013.

7. Sun J, Tu M, Han B, Xue X, et al: Generation and characterization of rabbit polyclonal antibodies against Vasohibin-2 for determination of its intracellular localization. Int J Oncol 43: 255-261, 2013.

8. Beresford MJ, Wilson GD and Makris A: Measuring proliferation in breast cancer: practicalities and applications. Breast Cancer Res 8: 216, 2006.

9. Lopez F, Belloc F, Lacombe F, Dumain P, Reiffers J, Bernard P and Boisseau MR: Modalities of synthesis of Ki67 antigen during the stimulation of lymphocytes. Cytometry 12: 42-49, 1991.
10. Verheijen R, Kuijpers HJ, Schlingemann RO, Boehmer AL, van Driel R, Brakenhoff GJ and Ramaekers FC: Ki-67 detects a nuclear matrix-associated proliferation-related antigen. I. Intracellular localization during interphase. J Cell Sci 92: 123-130, 1989.

11. Heidebrecht HJ, Buck F, Haas K, Wacker HH and Parwaresch R: Monoclonal antibodies $\mathrm{Ki}-\mathrm{S} 3$ and $\mathrm{Ki}-\mathrm{S} 5$ yield new data on the 'Ki-67' proteins. Cell Prolif 29: 413-425, 1996.

12. Allred DC, Mohsin SK and Fuqua SA: Histological and biological evolution of human premalignant breast disease. Endocr Relat Cancer 8: 47-61, 2001.

13. Rudas M, Neumayer R, Gnant MF, Mittelböck M, Jakesz R and Reiner A: p53 protein expression, cell proliferation and steroid hormone receptors in ductal and lobular in situ carcinomas of the breast. Eur J Cancer 33: 39-44, 1997.

14. Shoker BS, Jarvis C, Davies MP, Iqbal M, Sibson DR and Sloane JP: Immunodetectable cyclin $\mathrm{D}(1)$ is associated with oestrogen receptor but not Ki67 in normal, cancerous and precancerous breast lesions. Br J Cancer 84: 1064-1069, 2001.

15. Kontzoglou K, Palla V, Karaolanis G, Karaiskos I, Alexiou I, Pateras I, Konstantoudakis K and Stamatakos M: Correlation between Ki67 and breast cancer prognosis. Oncology 84: 219-225, 2013.

16. Sporn MB and Todaro GJ: Autocrine secretion and malignant transformation of cells. N Engl J Med 303: 878-880, 1980.

17. Witsch E, Sela M and Yarden Y: Roles for growth factors in cancer progression. Physiology (Bethesda) 25: 85-101, 2010.

18. Chandler LA, Sosnowski BA, Greenlees L, Aukerman SL, Baird A and Pierce GF: Prevalent expression of fibroblast growth factor (FGF) receptors and FGF2 in human tumor cell lines. Int J Cancer 81: 451-458, 1999.

19. Yin T, Cho SJ and Chen X: RNPC1, an RNA-binding protein and a p53 target, regulates macrophage inhibitory cytokine-1 (MIC-1) expression through mRNA stability. J Biol Chem 288: 23680-23686, 2013.

20. Chitnis MM, Yuen JS, Protheroe AS, Pollak M and Macaulay VM: The type 1 insulin-like growth factor receptor pathway. Clin Cancer Res 14: 6364-6370, 2008

21. Bach LA, Fu P and Yang Z: Insulin-like growth factor-binding protein-6 and cancer. Clin Sci (Lond) 124: 215-229, 2013.

22. Rocha RL, Hilsenbeck SG, Jackson JG, Lee AV, Figueroa JA and Yee D: Correlation of insulin-like growth factor-binding protein-3 messenger RNA with protein expression in primary breast cancer tissues: detection of higher levels in tumors with poor prognostic features. J Natl Cancer Inst 88: 601-606, 1996.

23. Rocha RL, Hilsenbeck SG, Jackson JG, VanDenBerg CL, Weng Cn, Lee AV and Yee D: Insulin-like growth factor binding protein-3 and insulin receptor substrate-1 in breast cancer: correlation with clinical parameters and disease-free survival. Clin Cancer Res 3: 103-109, 1997

24. Natsuizaka M, Kinugasa H, Kagawa S, Whelan KA, et al: IGFBP3 promotes esophageal cancer growth by suppressing oxidative stress in hypoxic tumor microenvironment. Am J Cancer Res 4: 29-41, 2014. 